\title{
Extracción de almidón del arroz de cebada por procesos físicos de separación
}

Extraction of starch from barley rice by physical separation processes

Extração de amido de arroz de cebada por processos de separação física

\author{
Hanníbal Lorenzo Brito Moína \\ hbrito@espoch.edu.ec \\ https://orcid.org/0000-0001-7536-857X
}

\author{
Iván Fernando Huacho Chávez \\ ivan.huacho@espoch.edu.ec \\ https://orcid.org/0000-0002-3144-3379
}

\author{
Adriana Isabel Rodríguez Basantes \\ adriana.rodriguez@espoch.edu.ec \\ https://orcid.org/0000-0002-2532-6504
}

\begin{abstract}
Escuela Superior Politécnica de Chimborazo (ESPOCH), Chimborozo, Ecuador
Artículo recibido 17 de febrero 2021 / Arbitrado y aceptado 24 de marzo 2021 / Publicado 04 de mayo 2021
\end{abstract}

\section{RESUMEN}

Este trabajo de investigación se desarrolló con la finalidad de obtener almidón de arroz de cebada (Hordeum vulgare L), para determinar cómo afecta el tamaño de partícula en el rendimiento de la misma, para lo cual, se procedió con el análisis organoléptico de la materia prima, posteriormente se redujo el tamaño para proceder a mezclar con agua aplicando el diseño factorial $2 \mathrm{k}$ considerando variables de proceso a la velocidad y tiempo con 3 repeticiones cada uno, de esta manera separar las macromoléculas, mediante la filtración y sedimentación hasta que el almidón se precipite en el fondo del recipiente, luego se separó del líquido y se secó a la temperatura ambiente, a continuación se homogeneizó hasta una granulometría uniforme, se pesó y determinó su rendimiento, luego se efectuó el análisis organoléptico, físico, químico y microbiológico, determinando que los parámetros de análisis se encuentran dentro de la norma para el uso como materia prima en otros procesos de manufactura. Los tratamientos fueron analizados estadísticamente mediante la prueba ANOVA y TUKEY para determinar la diferencia significativa entre los mismos, además de definir que el mejor tratamiento es el 4 con el 38,20 $\%$ de rendimiento.

Palabras clave: Arroz de cebada; Separación; Filtración;Sedimentación; Almidón

\section{ABSTRACT}

This research work was developed in order to obtain starch from barley rice (Hordeum vulgare L), to determine how particle size affects particle performance, for which, the organoleptic analysis of the raw material was carried out, later the size was reduced to proceed to mix with water applying the $2 \mathrm{k}$ factorial design considering process variables at speed and time with 3 repetitions each, in this way separate the macromolecules, by filtration and sedimentation until the starch precipitates at the bottom of the container, then separated from the liquid and dried at room temperature, then it was homogenized until a uniform granulometry, it was weighed and its performance determined, then the organoleptic analysis was carried out, physical, chemical and microbiological, determining that the analysis parameters are within the norm for use as raw material in other manufacturing processes. The treatments were statistically analyzed using the ANOVA and TUKEY tests to determine the significant difference between them, in addition to defining that the best treatment is 4 with 38,20 $\%$ yield.

Key words: Barley rice; Separation; Filtration; Sedimentation; Starch

\section{RESUMO}

Este trabalho de pesquisa foi desenvolvido como objetivo de obter amido de arroz de cevada (Hordeum vulgare L), para determinar como - tamanho da partícula afeta o desempenho da partícula, para o qual, procedemos com a análise organoléptica da matéria-prima, posteriormente o tamanho foi reduzido para proceder à mistura com água aplicando o planejamento fatorial $2 \mathrm{k}$ considerando variáveis de processo em velocidade e tempo com 3 repetições cada, desta forma, separar as macromoléculas, por filtração e sedimentação até que o amido precipite no fundo do recipiente, em seguida, separado do líquido e seco à temperatura ambiente, então foi homogeneizado até uma granulometria uniforme, seu desempenho foi pesado e determinado, em seguida, foi realizada a análise organoléptica, física, química e microbiológica, determinar se os parâmetros de análise estão dentro da norma para uso como matéria-prima em outros processos de fabricação. Os tratamentos foram analisados estatisticamente usando os testes ANOVA e TUKEY para determinar a diferença significativa entre eles, además de definir que el mejor tratamiento es el 4 con el 38,20\% de rendimiento.

Palavras-chave: Arroz de cevada; Separação; Filtração; Sedimentação; Amido 


\section{INTRODUCCIÓN}

El arroz de cebada se la obtiene, mediante un proceso de pelado y molido del Hordeum vulgare L. (1), que es una planta diploide, monoica y autógama (2), que pertenece a la familia de las gramíneas que es originaria del Asia y a nivel mundial ocupa el $9,4 \%$ de la superficie total de cereales cultivados, contribuyendo con un 7,8 \% de la producción total, en el Ecuador es uno de los cultivos más importantes de la sierra, la provincia de Chimborazo tiene la mayor área consagrada a este cultivo con 18000 ha de las 48000 ha que se producen en el país, le sigue Cotopaxi con 10 000 ha (3). Este cereal es rico en nutrientes por su alto contenido de carbohidratos (almidón), fibra dietética ( $\beta$-glucano polímero de glucosa que es inocuo, es muy utilizado en la industria de alimentos) (3), tiene concentración moderada de proteínas, es una buena fuente de fósforo, potasio, lípidos, compuestos nitrogenados, vitaminas y sales minerales. Por este motivo, este cereal en una variedad de formas como cebada perlada, harinas, copos, semolina y malta, que es muy utilizada para la producción de etanol, así como, múltiples usos y aplicaciones en el campo industrial (4).

Por lo expuesto, la presente investigación se enfoca en la producción de uno de los derivados del arroz de cebada como lo es la macromolécula de almidón que se encuentra compuesto por polímeros distintos de glucosa, como la amilosa (25-35\%) y la amilopectina (65$75 \%$ ). Es el glúcido de reserva de la mayoría de los vegetales (5).

Algunos métodos de modificación en su estructura química han permitido generar innumerables aplicaciones que se han visto beneficiadas con la obtención de almidones modificados (6) que son constituyentes fundamentales en diversos productos usados en la industria (7). Este estudio se basa específicamente en la utilización de un procedimiento físico sin cambios químicos para la separación de las macromoléculas de almidón en base a la utilización de Operaciones Unitarias (8) como agitación y mezcla (8), filtración, sedimentación, secado, reducción de tamaño y tamizado, aplicando el diseño experimental 2 factorial $k$, con 4 tratamientos y 4 repeticiones cada uno para determinar el mejor rendimiento de extracción del almidón (9) a partir del arroz de cebada, además se realizó el análisis estadístico para determinar si existieron diferencias significativas entre los tratamientos.

Se procedió con la caracterización del almidón obtenido para verificar el cumplimiento de la norma para su uso como materia prima en alimentos (10).

La cantidad de almidón extraído a partir de 200 gramos de materia prima en $750 \mathrm{~mL}$ de agua, para las tres repeticiones de cada tratamiento fue de 144,33 gramos para el tratamiento uno 106,02 gramos para el tratamiento dos 110 gramos para el tratamiento tres y 230 gramos para el tratamiento cuatro, obteniendo como rendimiento para el tratamiento uno $24,06 \%$ para el tratamiento dos $17,67 \%$ para el tratamiento tres $18,33 \%$ y para el cuarto tratamiento $38,33 \%$.

\section{MATERIALES Y MÉTODOS}

Se procedió con la recepción la materia prima (Hordeum vulgare L.), luego se 
analizó sus características físicas, químicas y microbiológicas, a continuación, se pesó 200 gramos para la preparación de la muestra, para lo cual, se trabajó mediante los métodos de extracción por molienda y por licuado.

En el primer método se sometió al proceso de reducción de tamaño mediante el uso de un molino marca "Corona" para grano a manivela, manteniendo el tornillo ajustado al $50 \%$ y $100 \%$ para obtener una granulometría gruesa y fina respectivamente, posteriormente se lavó con abundante agua con la finalidad de separar las macromoléculas de almidón, la mezcla fue filtrada con un filtro de vacío, el líquido separado fue llevado a un recipiente para su correspondiente sedimentación por un período de 6 horas, hasta que el almidón precipite al fondo del recipiente y así hacer más fácil su extracción, a continuación se eliminó el agua sobrenadante, los sólidos se secaron a la temperatura ambiente por el lapso de 12 horas, las escamas se llevaron a un molino de bolas, finalmente se separó en un tamiz de 38 um, para obtener una granulometría homogénea del almidón.

El segundo método se lo realizó mediante el proceso de trituración con la ayuda de una licuadora, "Oster" 3 velocidades 4655, con valores de velocidad mínima y máxima de 6800 RPM y 25000 RPM, respectivamente y una capacidad de 1,25 L. Como primer paso se introdujo 200 gramos de arroz de cebada en la jarra de vidrio y se agregó agua hasta un volumen de $750 \mathrm{~mL}$. Posteriormente se licuó el grano por 2 minutos variando la velocidad de giro (11) para las posteriores repeticiones.
Como siguiente paso, se lavó la materia prima triturada con agua y se trasladó este contenido a otro recipiente haciéndolo pasar por un tamiz para retener los gránulos de arroz de cebada. El almidón (12) obtenido, disuelto en agua, pasa juntamente con esta hacia el recipiente, se dejó en reposo con el contenido durante 8 horas para que el almidón (13) sedimente al fondo de este. Cumplido este lapso de tiempo, se separó el sobrenadante del sedimento y este último se dejó secar a temperatura ambiente (14) por 12 horas. El resultado final fue la obtención de un polvo amarillento, almidón (15) deseado, este proceso se lo realiza por cuadruplicado y para cada uno de los tratamientos.

El almidón de arroz de cebada obtenido por los 2 métodos, fue analizado por métodos físicos, químicos y microbiológicos (16) en los cuales se detallan los valores de amilosa, amilopectina (17), humedad, ceniza, pH, fibras, proteínas, entre otros (18).

Con los datos obtenidos de los pesos se determinó el rendimiento mediante la relación de peso seco de almidón obtenido (19) y peso del arroz de cebada, y finalmente para identificar si existen diferencias significativas entre los tratamientos se efectuó el cálculo de la varianza por el método de ANOVA y la prueba TUKEY.

\section{RESULTADOS DISCUSIÓN}

Se realiza el análisis de 4 variedades de arroz de cebada como la Cañicapa (forrajera), Metcalfe (maltera), dorada y Pacha para determinar las mejores características físicas de cada una de ellas de acuerdo al peso, 
longitud y diámetro de los granos, valores que se muestran en el Figura 1, con lo cual, se determina el efecto que tiene el tamaño del grano en su peso, con valores que van de 19,6 a 23,1 mg; su longitud de 6,6 a 7,1 $\mathrm{mm}$ y el diámetro de 2,7 a $3 \mathrm{~mm}$; valores que según el estudio realizado por Días, (20) se encuentran en los rangos establecidos para un grano de arroz de cebada que va de 3 a 8 $\mathrm{mm}$ de longitud; mismos que dependerán de las características del cultivo y su naturaleza, en esta investigación se partió con 200 gramos y una media aritmética a los tratamientos en cuanto a su longitud en promedio de grano fue de $6,7 \mathrm{~mm}$ considerable para su uso y en cuanto a su diámetro promedio es de $2,8 \mathrm{~mm}$ valor que según Hernández (21) se encuentra en el rango identificado que va de $1,5-4,00 \mathrm{~mm}$; valores que indican que el grano de cebada se encuentra sano y adecuado para la extracción del almidón.
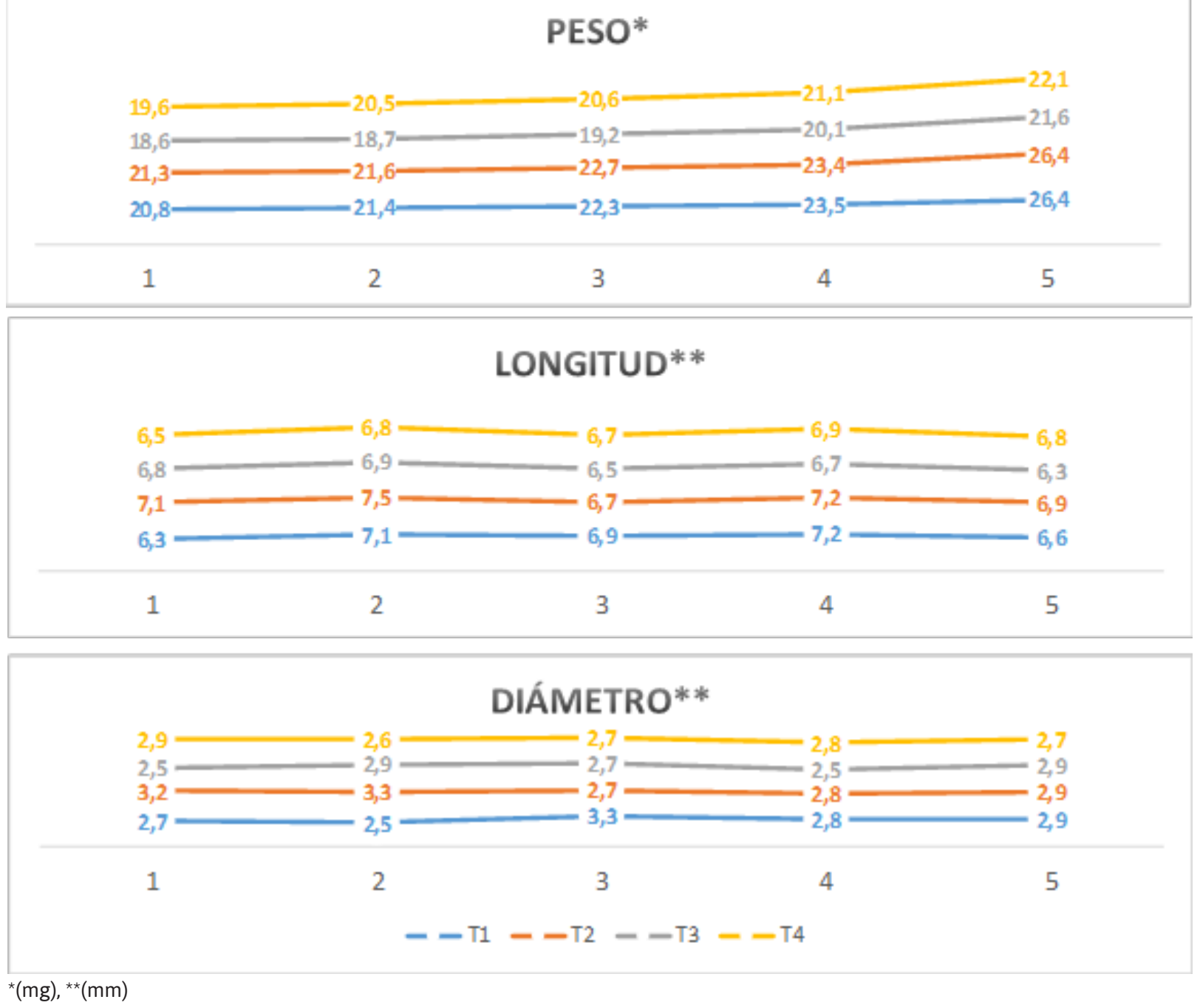

Figura 1. Características Físicas del grano de Arroz de Cebada. Fuente: Brito H., Laboratorio de Investigación, Facultad de Ciencias, ESPOCH, 2021. 
Tabla 1. Caracterización organoléptica del arroz de cebada y su almidón.

\begin{tabular}{rlll}
\hline No. & ENSAYOS & ARROZ DE CEBADA & ALMIDÓN ARROZ DE CEBADA \\
\hline 1 & Color & Café - Típico & Café claro \\
2 & Olor & Característico & Característico \\
3 & Sabor & Agradable, Dulce - Característico & Agradable - Característico \\
4 & Textura & Grano Rugoso seco & Harina - Polvo \\
5 & Forma & Circular - Ovalada & Esférico lenticular \\
\hline
\end{tabular}

Fuente: Brito H., Laboratorio de Investigación, Facultad de Ciencias, ESPOCH, 2021.

Se realizó el análisis organoléptico de los gránulos del arroz de cebada (Tabla 1 ), en el cual, se consideraron cinco parámetros como el color, olor, sabor, Textura y forma; en cada variable se dio dos sub ensayos de manera sensorial determinando que presenta en la Tabla 1 con un color café, olor característico, sabor agradable dulce, con una forma circular ovalada y en estado rugoso seco como es lo típico de un grano. Según Brito (9) la ausencia de alteraciones de olor y color de los granos es una señal de que no existe contaminación por hongos o insectos; sensorialmente, la mayoría de las variedades presentaron un olor y color característico del grano sano y seco, concordando con el análisis efectuado. En lo relacionado al análisis organoléptico del almidón de arroz de cebada se consideraron parámetros de color, olor, sabor y textura; con dos sub análisis determinando que tiene un color café claro, el olor característico, sabor agradable, con una textura y forma esférica lenticular, parámetros que se encuentran de acuerdo a lo indicado por Agama (22); producto que puede ser utilizado en la fabricación de diferentes bio-productos, como azúcares fermentables para la obtención de ácido láctico, que en la actualidad es de gran importancia en la elaboración de polímeros y solventes biodegradables.

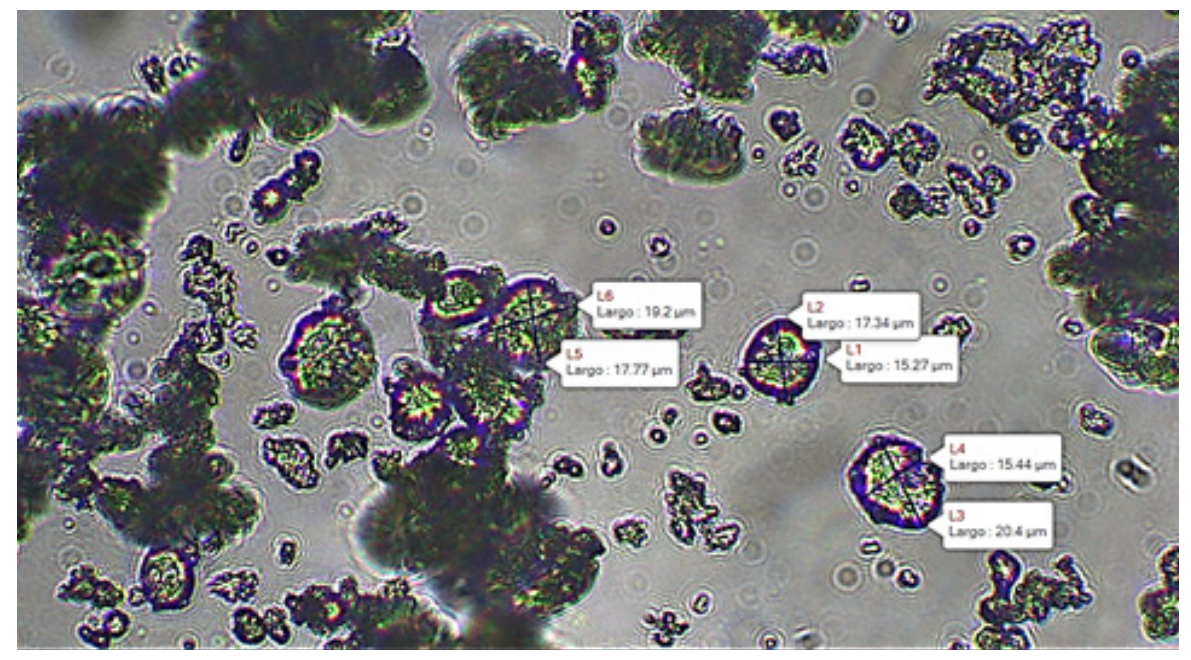

Figura 1. Forma del gránulo de almidón del arroz de cebada. Fuente: Brito H., Laboratorio de Investigación, Facultad de Ciencias, ESPOCH, 2021. 
Las macromoléculas de almidón fueron analizadas en el microscopio como se indica en la Figura 2 determinando que la forma del gránulo de almidón del arroz de cebada es de tipo lenticular de 17,57 um en promedio valor que se encuentra en los rangos establecidos en el estudio de Cárdenas (23).

Tabla 2. Análisis físico, químico y midrobiológico del almidón de arroz de cebada.

\begin{tabular}{llccrl}
\hline No. & \multicolumn{1}{c}{ ENSAYOS } & UNIDAD & ALMIDÓN & ESTÁNDAR & NORMA \\
\hline 1 & Humedad & $\%$ & 1,70 & $\leq 13$ & NMX-F-382-1986 \\
2 & Ceniza & $\%$ & 0,23 & $\leq 0,12$ & $\begin{array}{l}\text { AOAC (Association of Official } \\
\text { Analytical Chemists) } 942.05\end{array}$ \\
3 & Solubilidad & $\%$ & 0,10 & Ligera opalencia & INEN 1456* \\
4 & pH & - & 6,21 & $6-7$ & INEN 1456 \\
5 & Temperatura de gelatinización & ${ }^{\circ} \mathrm{C}$ & 74,67 & 78 & CÁRDENAS, 2018 \\
6 & Amilosa & $\%$ & 32,7 & $20-40$ & INIAP \\
7 & Amilopectina & $\%$ & 67,3 & $60-80$ & INIAP \\
8 & Hongos & UFC/g & 621,3 & $1000-5000$ & FAO 2007 \\
9 & Mohos y Levaduras & UFC/g & 1372,4 & $1000-5000$ & FAO 2007 \\
10 & Coliformes Totales & UFC/g & 0,667 & Ausencia & FAO 2007 \\
\hline
\end{tabular}

Fuente: Brito H., Laboratorio de Investigación, Facultad de Ciencias, ESPOCH, 2021.

Luego de realizar la caracterización físico química y microbiológica del almidón obtenido como se indica en la Tabla 2 , se determina que el contenido de humedad de 1,70\% se encuentra dentro del rango establecido en la norma NMX-F-382-1986, en cuanto al porcentaje de cenizas se verifica que es casi el doble al valor establecido en la norma AOAC (Association of Official Analytical Chemists), el pH está en el rango establecido por la norma INEN 1456 y la temperatura de gelatinización de $74,67^{\circ} \mathrm{C}$ de acuerdo a lo establecido en el estudio de Cárdenas (23). Además el análisis de amilosa y amilopectina obtenidos en el almidón de arroz de cebada son de $32,7 \%$ y 67,3 $\%$ respectivamente valores que van a mejorar las propiedades mecánicas de las láminas de bioplástico, en cuanto a mohos y levaduras (24) con un valor de 1372,4 UFC/g, están dentro del rango permitido en la norma peruana RM N615-2003 SAJDM que establece los criterios microbiológicos de calidad sanitaria e inocuidad para los alimentos y bebidas de consumo humano dando un óptimo resultado para su posterior uso en la elaboración de láminas de plástico. 
Tabla 3. Rendimiento del almidón de arroz de cebada.

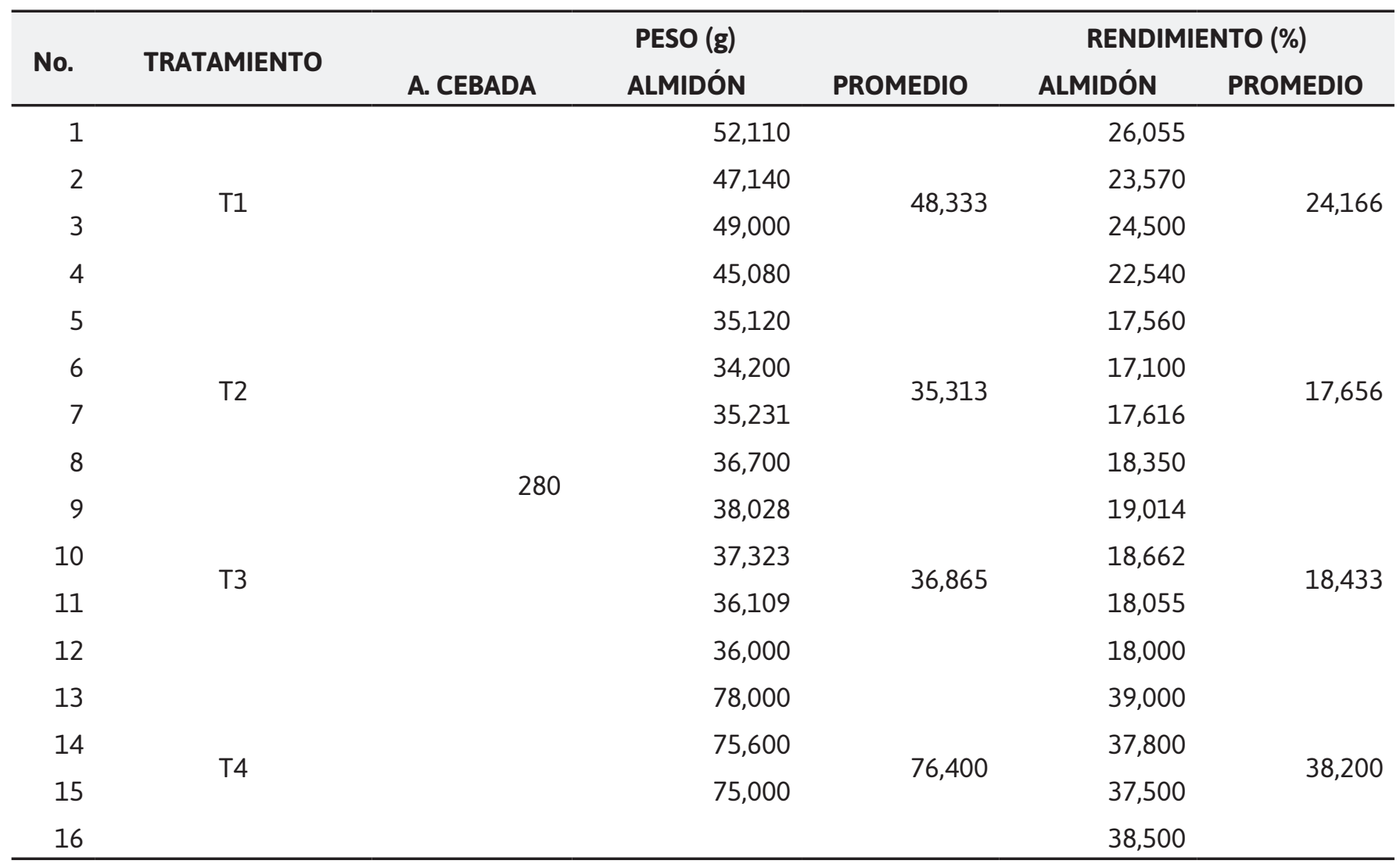

Fuente: Brito H., Laboratorio de Investigación, Facultad de Ciencias, ESPOCH, 2021.

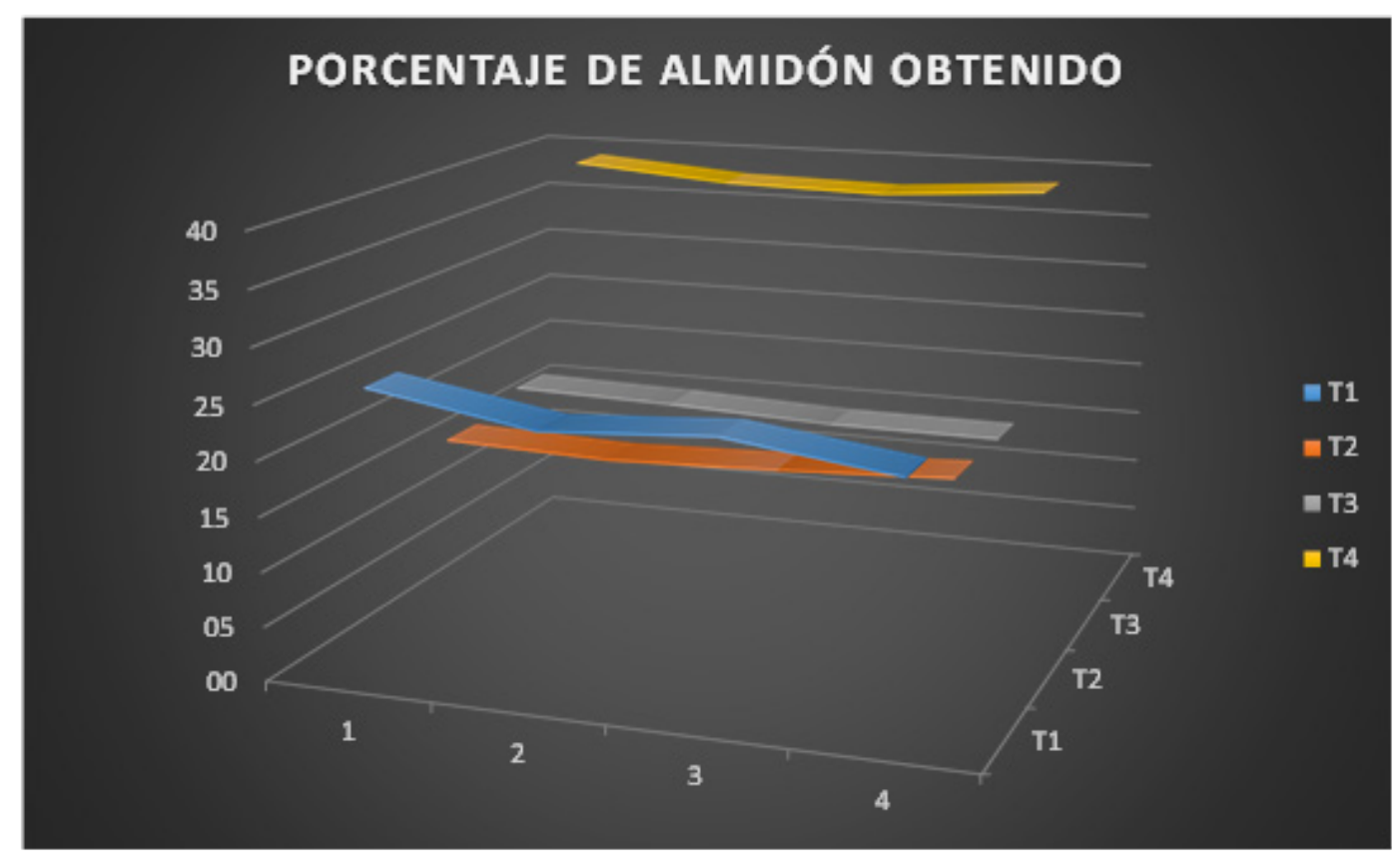

Gráfico 1. Porcentaje de almidón obtenido. Fuente: Brito H., Laboratorio de Investigación, Facultad de Ciencias, ESPOCH, 2021. 
Luego de establecer el peso de las repeticiones de cada uno de los tratamientos se obtuvo el rendimiento (Tabla 3) determinando que son diferentes debido al uso de dos equipos el primero un molino y el segundo una licuadora separando las macromoléculas del arroz de cebada, determinando que el rendimiento aumenta con la disminución del área de superficie de la materia prima, así como también, el tiempo y la velocidad de los equipos utilizados que influyen directamente, identificando que el tratamiento 4 con el 38,2 $\%$ tiene mejor rendimiento, esto se debe, a la disminución rápida del área superficial.

Tabla 4. Análisis de la Varianza en el rendimiento del almidón de arroz de cebada.

\begin{tabular}{cccccccc}
\hline $\begin{array}{c}\text { Origen de las } \\
\text { variaciones }\end{array}$ & $\begin{array}{c}\text { Suma de } \\
\text { cuadrados }\end{array}$ & $\begin{array}{c}\text { Grados de } \\
\text { libertad }\end{array}$ & $\begin{array}{c}\text { Promedio de } \\
\text { los cuadrados }\end{array}$ & F & Probabilidad & $\begin{array}{c}\text { Valor crítico } \\
\text { para F }\end{array}$ \\
\hline Muestra & 219,3323975 & 1 & 219,3323975 & 274,705753 & $1,2351 \mathrm{E}-09$ & 4,747225347 \\
Columnas & 175,7646206 & 1 & 175,7646206 & 220,138717 & $4,4031 \mathrm{E}-09$ & 4,747225347 \\
Interacción & 690,5004369 & 1 & 690,5004369 & 864,826377 & $1,491 \mathrm{E}-12$ & 4,747225347 \\
Dentro del grupo & 9,581119938 & 12 & 0,798426661 & & & \\
Total & 1095,178575 & 15 & & & & \\
\hline
\end{tabular}

Fuente: Brito H., Laboratorio de Investigación, Facultad de Ciencias, ESPOCH, 2021.

Los datos obtenidos del rendimiento en cada uno de los tratamientos fueron analizados estadísticamente con el test estadístico Anova (Tabla 4) con un valor del $5 \%$ de error se determina que existen diferencias significativas entre los tratamientos, motivo por el cual, fue necesario identificar entre los tratamientos su diferencia significativa, para lo cual, se utilizó el test de Tukey (Tabla 5) determinando que el valor de HSD es de 1,876 mismo que al ser comparado con la diferencia de los promedios de los tratamientos se identificó que entre los tratamientos T2 - T3 no existe diferencia significativa, en cambio con el resto de tratamientos si tienen diferencia significativa.

Tabla 5. Análisis del test de Tukey.

\begin{tabular}{ccccc}
\hline & T1 & T2 & T3 & T4 \\
\hline T1 & & 6.5 & 5,7 & 14,0 \\
T2 & & & 0,8 & 20,5 \\
T3 & & & & 19,8 \\
T4 & & & & \\
\hline
\end{tabular}

Fuente: Brito H., Laboratorio de Investigación, Facultad de Ciencias, ESPOCH, 2021. 


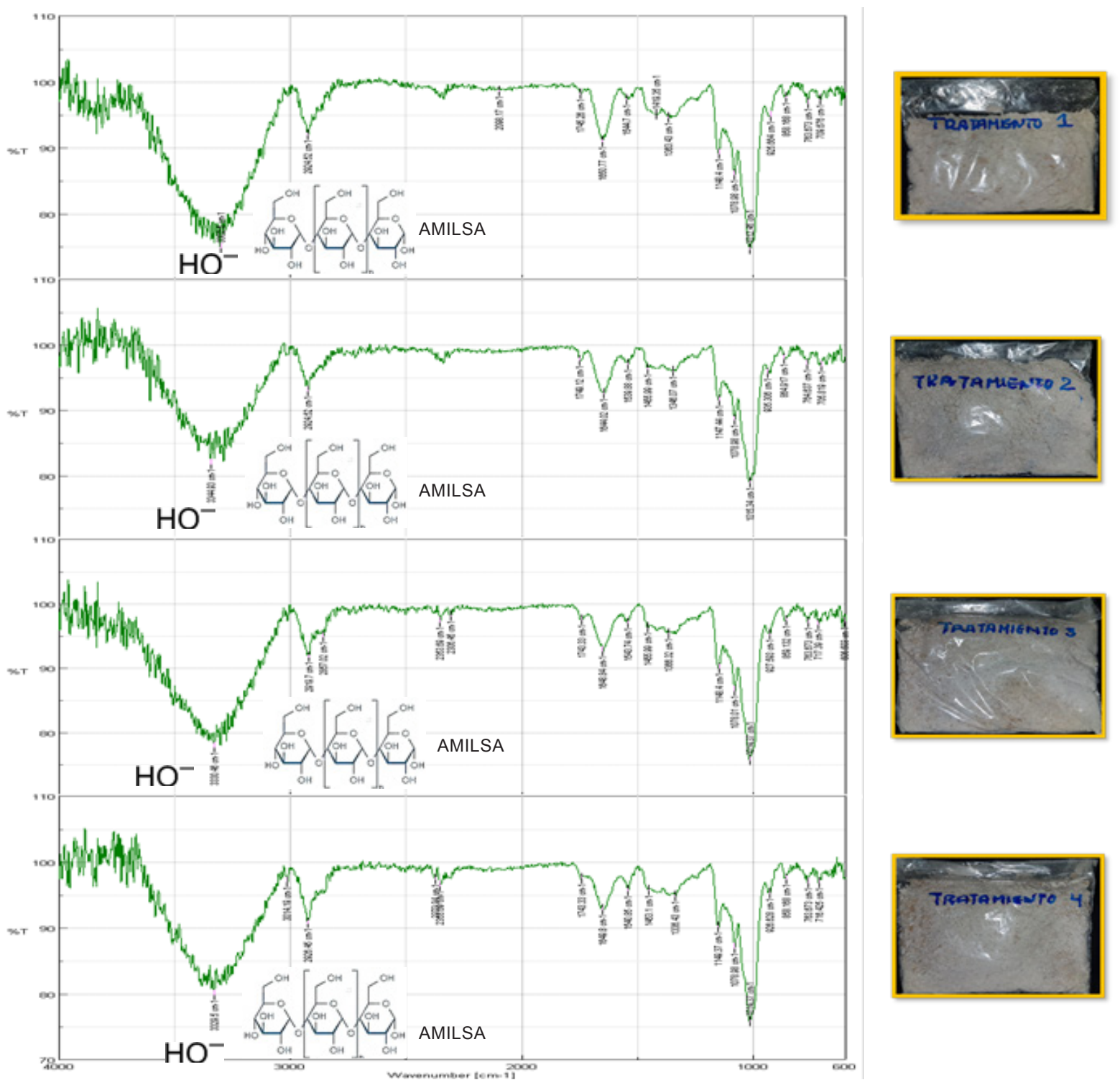

Gráfico 2. IR Almidón del Arroz de Cebada Tratamientos. Fuente: Brito H., Laboratorio de Instrumental, Facultad de Ciencias, ESPOCH, 2021.

Tabla 6. Presencia de grupos funcionales en el almidón del arroz de cebada.

\begin{tabular}{cccl}
\hline No. & BANDA $\left(\mathbf{c m}^{-1}\right)$ & GRUPO FUNCIONAL & \multicolumn{1}{c}{ OBSERVACIÓN } \\
\hline 1 & $3302,5-3344,93$ & $-\mathrm{OH}$ & Vibración de estiramiento del enlace $-\mathrm{OH}$ \\
2 & $2919,7-2926,45$ & $-\mathrm{CH} 2$ & Vibración de estiramiento del enlace $-\mathrm{CH} 2$ \\
3 & $1147,44-1149,37$ & $\mathrm{C}-\mathrm{O}-\mathrm{C}$ & Vibración de estiramiento antisimétrico del enlace C-O-C \\
4 & $1012,45-1015,34$ & $\mathrm{C}-\mathrm{O}$ & Vibración de estiramiento del enlace C-O \\
\hline
\end{tabular}

Fuente: Brito H., Laboratorio de Investigación, Facultad de Ciencias, ESPOCH, 2021. 
Se realizó el análisis Infra rojo a los 4 tratamientos del almidón de arroz de cebada como se presenta en la Tabla 6, determinando la presencia del grupo funcional $\mathrm{OH}^{-}$en el rango de $3302,5 \mathrm{~cm}^{-1}$ para el tratamiento 1 ; $3344,93 \mathrm{~cm}^{-1}$ del tratamiento 2; 3330,46 $\mathrm{cm}^{-1}$ tratamiento 3 y $3329,5 \mathrm{~cm}^{-1}$ del tratamiento 4 , evidenciando la presencia de amilosa molécula lineal de almidón que está constituida por muchos anillos de glucosa unidos entre sí para formar largas moléculas que no tienen ramificaciones; además se encuentra la presencia de grupos funcionales en el rango de 2919,7 a 2926,45 generando la vibración de estiramiento del enlace-CH2; de 1147,44 a 1149,37 con la Vibración de estiramiento anti simétrico del enlace C-O-C y finalmente de 1012,45-1015,34 Vibración de estiramiento del enlace $\mathrm{C}-\mathrm{O}$, componentes que se encuentran en la estructura de la amilosa y amilopectina macromoléculas presentes en el almidón.

Las características físicas del Arroz de cebada varían de 19,6 a 23,1 mg de peso; longitud de 6,6 a $7,1 \mathrm{~mm}$ y el diámetro de 2,7 a $3 \mathrm{~mm}$.

El análisis organoléptico del arroz de cebada tiene como características un color café, olor característico, sabor agradable dulce, con una forma circular ovalada y en estado rugoso; en cambio para el almidón es de color café claro, el olor característico, sabor agradable, con una textura y forma esférica lenticular.

De acuerdo a las propiedades físico químicas obtenidas se determina que el contenido de humedad es de 1,70\%; ceniza $0,23 \%$; solubilidad de 0,10\%; $\mathrm{pH}$ de 6,21, temperatura de gelatinización de 74,67; amilosa de $32,7 \%$ y amilopectina de $67,3 \%$.

En el análisis microbiológico se obtuvo el valor para hongos de 621 UFC/g; Mohos y levaduras de 1372,4 UFC/g y ausencia de coliformes totales.

Del análisis infra rojo se determina la presencia del grupo funcional $\mathrm{OH}^{-}$en el rango de $3302,5 \mathrm{~cm}^{-1}$ para el tratamiento 1 ; $3344,93 \mathrm{~cm}^{-1}$ del tratamiento 2; $3330,46 \mathrm{~cm}^{-1}$ tratamiento 3 y 3329,5 cm-1 del tratamiento 4 .

El mejor rendimiento es del tratamiento 4 con el 38,2 \%.

En el análisis estadístico se determina que los tratamientos T2 - T3 no tienen diferencia significativa y el resto de tratamiento si presentan diferencia significativa.

\section{Discusión}

Luego de obtener el almidón por los dos equipos y con los 4 tratamientos, se determinó que el tratamiento cuatro con el 38,2 \% tiene mejor rendimiento, valor que se encuentra en los objetivos planificados para este estudio.

Luego del análisis estadístico se determinó que no existe diferencia significativa entre los tratamientos T2 - T3, es decir, que los rendimientos obtenidos son similares, por lo tanto, se puede trabajar con cualquiera de ellos y se acepta la hipótesis nula, lo que no se puede decir del resto de tratamientos que si tienen diferencia significativa entre ellos.

\section{CONCLUSIONES}

La obtención de biopolímero natural procedente del arroz de cebada tiene como características fundamentales una forma 
del gránulo de almidón es de tipo redondo lenticular con un promedio de 17,57 um, valores que se encuentran en lo establecido en el estudio de Chuiza, (25).

Los valores obtenidos en el análisis de amilosa (estructura lineal) y amilopectina (estructura ramificada) obtenido en el almidón de arroz de cebada son de 32,7 \% y $67,3 \%$ respectivamente, identificando que el biopolímero natural se encuentra en el rango establecido por la FAO (26).

\section{REFERENCIAS BIBLIOGRÁFICAS}

1. Faliana C, Romina $R$, Francisco $P$, Alma R. Caracterización Física y Química Proximal de Paja, Grano y almidón de cebada. BioTecnología. 2012;16(3).

2. López P, Guzmán F, Santos E, Prieto F, Román A. Evaluación de la calidad física de diferentes variedades de cebada (HORDEUM SATIVUM JESs) cultivadas en los estados de Hidalgo Y Tlaxcala, México. Revsta chilena de nutrición. 2005 Noviembre; 32(3).

3. INIAP. Instituto Nacional de Investigaciones agropecuarias. [Online]. [cited 2021 abril 16. Available from: https://www.google.com/search?q= cultivo+de+cebada+en+ecuador\&source =hp\&tei=bHEWYb2JKPGdwbkPxbSdqAo\&iflsig $=A \mid N F C$ b Y A A A A A Y R Z fLQbkPSdk7mupIrREk_2WAUHgyf7\&o $=c u l t i v o+d e+c e v a d a \& g s+l c p=$ Cgdnd3Mtd2l6EAEYATIECAAQCjIE C A A Q C J IE CA A Q C J IE C A A Q C J IE CAAQCjIECAAQCjIECAAQCjIEC

4. Pizarro CS, Ronco MAM, Gotteland RM. B-glucanos: ¿qué tipos existen y cuáles son sus beneficios en la salud? B-glucans: what types exist and what are their health benefits? Rev. Chil. nutr. 2014;41(4):439-446. doi: http://dx.doi. org/10.4067/S0717-75182014000400014.

5. Castellanos J. Determinación de propiedades fisicoquímicas y características microbiológicas en muestras de materias primas y productos elaborados. [Online]; 2010 [cited 20200620. Available from: HYPERLINK http://bdigital. ula.ve/storage/pdftesis/pregrado/tde arquivos/33/TDE-2012-09-19T03:18:11Z-1655/ Publico/castellanosjesus.pdf.

6. Agama E, Bello L, Zamudio P, Rodriguez S. Efecto del bajo y alto grado de acetilación en las características morfológicas, fisicoquímicas y estructurales del almidón de cebada. Ciencia y tecnología de alimentos. 2010

7. Gupta M, Singh A, Dutt A. Propiedades morfológicas, térmicas, de pegado y reológicas del almidón de cebada y sus mezclas. International Journal of Food Properties. 2009.

8. Ramírez $T$, Gómez A. Efecto del solvente acidificado sobre las propiedades funcionales del almidón. Congreso Nacional de Ciencia y Tecnología de Alimentos. 2010 Mayo.

9. Brito H. Texto Básico de Operaciones Unitarias II Riobamba: Docucentro ESPOCH; 2001.

10. Brito $H$, et al. Diseño de un proceso de producción industrial de almidón a partir de mashua (Tropaeolum tuberosum). La Ciencia al Servicio de la Salud y la Nutrición. 2019;: p. 202-209.

11. Coello G. Elaboración y valoración nutricional de tres productos alternativos a base de cebada para escolares del proyecto RUNA KAWSAY. In. Riobamba; 2010:124.

12. Robalino P, Heredia S, Chango G, Flores L, Salazar K, Brito H. Diseño de un proceso de producción industrial de almidón a partir de mashua. La Ciencia al Servicio de la Salud y la Nutrición. 2019 Julio; 10.

13. Meneses J, Corrales C, Valencia M. Síntesis y caracterización de un polímero biodegradable a partir de almidon de yuca. Escuela de Ingeniería de Antioquia. 2007 Diciembre;(8).

14. Puchicela. Extracción de Almidón de Arroz. Sangolquí: Universidad de las Fuerzas Armadas (ESPE), Departamento de Ciencias de la Vida y la agricultura; 2015. 
15. Brito H. Texto Básico de Operaciones Unitarias III Riobamba, Ecuador: Documento, ESPOCH; 2001.

16. Pérez E, Lares M, Gonzáles Z, Tovar J. Production and Characterization of Cassava (Manihot esculenta CRANTZ) Flour using different thermal treatments: interciencia; 2007.

17. BonettiC. Análisis del control microbiológico de los cereales en el momento de ingreso a Venezuela. INVESTIGA UNIPAP. 2013.

18. Angeles $P$. Diseño de un proceso industrial para obtener plástico biodegradable (TPS) a partir de almidón de yuca manihot sculenta Lambayeque: Universidad Nacional Pedro Luis Gallo; 2015.

19. Brito H, Borja D, Chango G. Obtaining yacon Flour (Smallanthus sonchifolius). 2019.

20. Waliszweski K, García Alvarado M, De la Cruz Medina J. Kinetics of enzymic hydroysisof cassava flour starch - optimization modelling: International journal of Food Science \& Technology; 2007.

21. Días Arca J, et al. Grado de molienda y utilización del alimento por conejos en crecimiento. Archivos de zootecnia. 1989; 38(140).
22. Hernández T. Estudio de la composición química de la cebada cultivada en Zapotlán. In.; 2006:100.

23. Agama Acevedo E, et al. Características del almidón de maíz y relación con las enzimas de su biosíntesis. Agrociencia. 2013;47(1).

24. Cárdenas Freire MA. Extracción del almidón a partir de residuos de banano (Musa paradisiaca) para la elaboración de un biopolímero Cuenca: Universidad Politécnica Salesiana; 2018.

25. Lozada L. Manual de Microbiología de los alimentos. [Online]. 2008. Available from: HYPERLINK http://www.unsa.edu.ar/biblio/ repositorio/malim $2007 / 8 \% 20$ granos $\% 20 y \% 20$ harinas.pdf.

26. Chuiza M, Rodríguez A, Brito H. Producción de láminas de plástico biodegradables a partir del almidón de arracacia xanthorrhiza. Ciencias técnicas y aplicadas. 2020 Junio; 6(2). 27. FAO. Guía técnica para producción y análisis de almidón de yuca Roma: FAO; 2007. 